\title{
Enhancing Fluoride: Clinical Human Studies of Alternatives or Boosters for Caries Management
}

\author{
Margherita Fontana \\ Department of Cariology, Restorative Sciences and Endodontics, University of Michigan School of Dentistry, \\ Ann Arbor, Mich., USA
}

\section{Key Words}

Amorphous calcium phosphate $\cdot$ Arginine $\cdot$ Calcium .

Casein phosphopeptide · Clinical trials · Fluoride .

Polyphenol $\cdot$ Remineralization $\cdot$ Xylitol

\begin{abstract}
Dental caries remains a major public health problem, especially for certain high-risk population groups. The goal of this study was to assess the evidence regarding strategies meant to be used as alternatives or booster/supplements to fluoride for caries prevention and management. Articles were selected for inclusion if they had a prospective longitudinal design, with a fluoride control arm, and were conducted in human subjects. Of the included studies, $7 / 18$ studies on calcium-based strategies favored the test product (the majority of studies included exposure of fluoride in all groups). All the arginine studies (8/8) included a combination of arginine and a calcium base, and concluded that this has the potential to significantly boost the performance of fluoride. The remaining included studies focused on the addition of microbial-related strategies to a fluoride-containing vehicle ( $2 x y$ litol studies and 1 study using a probiotic milk), and all favored the combination as a booster to fluoride. Thus, the current study did not identify evidence for any strategy to
\end{abstract}

\section{KARGER}

E-Mail karger@karger.com www.karger.com/cre

\section{C) 2016 The Author(s) \\ Published by S. Karger AG, Basel 0008-6568/16/0507-0022\$39.50/0}

This article is licensed under the Creative Commons AttributionNonCommercial-NoDerivatives 4.0 International License (CC BYNC-ND) (http://www.karger.com/Services/OpenAccessLicense) Usage and distribution for commercial purposes as well as any distribution of modified material requires written permission. effectively be used as a substitute or alternative to fluoride, but identified some consistent evidence derived from the use of prebiotic strategies (primarily from use of arginine combined with calcium) to support their potential use to boost the mechanism of action of fluoride. Thus, fluoridebased strategies remain the standard for caries prevention and management, with some evidence that boosting the effects of fluoride by the use of prebiotic strategies is a promising possibility.

(c) 2016 The Author(s)

Published by S. Karger AG, Basel

In spite of the significant reduction in caries prevalence in many parts of the world, dental caries remains a major public health problem affecting people of all ages. Furthermore, the disease is not equally distributed, with multiple population groups at increased risk. Existing caries management strategies include, for example, the promotion of a healthy diet, including a low frequency of exposure to fermentable carbohydrates, and frequent oral hygiene with fluoride-containing toothpaste. However, even with regular fluoride use, caries can still develop if caries risk factors are present. In addition, as the population ages, more root surfaces are exposed, and the potential for root caries increases, which may require higher 
levels of fluoride to prevent caries. These challenges support the need for continued research into cost-effective strategies for caries prevention and management that could work as a booster/supplement or as an alternative to fluoride [ten Cate, 2012]. Desired benefits from the use of boosters or alternatives to fluoride include enhanced efficacy of fluoride without the need to increase the concentration (e.g. enhanced remineralization and/or control of dental plaque), decreased risks associated with fluoride use (fluorosis) and toxicity, and expanded access to high caries risk populations (e.g. children at risk of fluorosis, patients with hyposalivation, root caries, etc.).

Remineralization is the process of incorporating calcium and phosphate into the demineralized tooth structure, resulting in net mineral gain [Cochrane et al., 2010]. Remineralization agents should deliver calcium and phosphate, or boost the remineralization potential of saliva, without any side effects [Amaechi and van Loveren, 2013]. Fluoride is a very effective strategy to control dental caries and aid in tooth remineralization [Marinho et al., 2002, 2003a, b, 2004a, b; Walsh et al., 2010; Benson et al., 2013], and is considered the gold standard for studying remineralization. The goal of this study was to assess the evidence derived from human clinical trials regarding strategies meant to be used as alternatives or booster/supplements to fluoride for caries prevention and management.

\section{Methods}

A broad search restricted to papers in the English language published between 1990 and January 2015 was carried out in PubMed. References included in pertinent systematic reviews were also hand searched. Reports in the gray literature (theses, etc.) were not included. MeSH and free terms used included the following: 'casein, casein phosphopeptide-amorphous calcium phosphate (CPP-ACP), amorphous calcium phosphate (ACP), calcium, Recaldent, MI Paste, Novamin, xylitol, probiotic, herbal, polyphenol, arginine, fluoride, clinical trial, humans, remineralization, cariostatic agent, toothpaste, mouthrinse, varnish, dental caries'. A comparison of fluoride used in multiple forms, such as sodium fluoride, sodium monofluorophosphate (MFP), stannous fluoride, etc. [Marinho et al., 2003b] was not the focus of the manuscript. Articles resulting from the search were selected for inclusion if they met the following criteria:

1 The study must have a prospective longitudinal design, with a fluoride control arm, be conducted in human subjects (in situ studies were excluded), and report an outcome measuring caries lesions (studies focusing only on erosion, tooth sensitivity, dry mouth relief, gingivitis, malodor, calculus, whitening, etc. were excluded). Trials that reported only surrogate end points (e.g. bacterial counts, $\mathrm{pH}$ values, calcium concentration, etc.) were not included.

Fluoride Boosters and/or Alternatives
2 The test intervention must have been used as a booster or as an alternative to fluoride. Strategies evaluated included calciumbased strategies (CPP-ACP, ACP, calcium-based compounds), prebiotic/probiotic strategies (xylitol, arginine, probiotics), herbal strategies (polyphenols) and any other strategy (e.g. biomimetic self-assembling peptides) resulting from a 'remineralization' search that was not fluoride. Chlorhexidine was not included in this search as it is a broad-spectrum antimicrobial to be used periodically, very different from the other strategies selected which, like fluoride, could be used daily for caries control depending on the vehicle and concentration used.

3 In addition, only therapies provided in toothpaste, cream, gels, varnishes or mouthwashes were included (chewing gum and lozenges were excluded, as control fluoride products in these vehicles are not commonly available around the world).

4 Essential data on interventions, study design, sample size, duration and results were extracted from selected studies. No formal quality assessments or grading were performed, but if the study had been included in a systematic review that had graded the study quality, this was reported.

\section{Results}

The search identified 142 publications. After removing duplicates, studies that did not meet the inclusion criteria based on abstract reviews, and reviews or systematic reviews, 60 publications were identified for further review. Finally, 28 publications were included, 18 associated with calcium-based strategies [9 for CPP-ACP, 3 for CPP-ACP plus fluoride, ACFP, 1 for casein derivatives with calcium phosphate (CD-CP), 1 for ACP, 1 for ACFP, 1 for $\mathrm{CaF}_{2}, 2$ for dicalcium phosphate dehydrate] and 10 with microbial-related strategies ( 8 for arginine, 2 for xylitol) - plus 1 using a milk vehicle for probiotic. No other strategies (e.g. biomimetic self-assembling peptides, polyphenols) met the inclusion criteria.

The majority of existing evidence is derived from the use of calcium-based strategies (table 1), especially CPP-ACP, and evidence is lacking for head-to-head comparisons of the various remineralization strategies. Of the included studies, 7/18 of the calcium-based strategies (meant to primarily enhance remineralization at the tooth level) favored the addition of the tested strategy compared to a fluoride arm (and yet the majority of these studies included exposure to background fluoride to all groups). Of the evidence for microbial-related strategies, the majority was derived from the use of arginine (tables 2 and 3). All the arginine studies (8/8) included a combination of arginine and a calcium base, and concluded that the addition of $1.5 \%$ arginine to toothpaste containing 1,450 ppm F (as MFP) with an insoluble calcium base has the potential to significantly boost the performance of fluoride-only toothpaste. The re-

Caries Res 2016;50(suppl 1):22-37 
Table 1. Calcium-based strategies versus fluoride as alternatives or boosters for clinical caries management

\begin{tabular}{|c|c|c|c|c|c|}
\hline Study & Test product & Subject characteristics & $\begin{array}{l}\text { Study design/ } \\
\text { outcome measure }\end{array}$ & Results & $\begin{array}{l}\text { Authors' conclusion } \\
\text { (reviewer comment) }\end{array}$ \\
\hline $\begin{array}{l}C P P-A C P \text { studies } \\
\text { Yetkiner et al. } \\
{[2014]}\end{array}$ & $\begin{array}{l}\text { CPP-ACP cream (Tooth } \\
\text { Mousse; GC) } 2 \times / \text { day after } \\
\text { brushing with } 1,450 \text { ppm F } \\
\text { toothpaste } \\
\begin{array}{l}\text { Fluoride toothpaste }(1,450 \\
\text { ppm F; Colgate) } 2 \times / \text { day }\end{array}\end{array}$ & $\begin{array}{l}\mathrm{n}=60 \\
\text { Children } 13 \pm 0.68 \text { years of } \\
\text { age (Turkey; at least } 1 \text { white } \\
\text { spot lesion; DMFS }=1.95 \pm \\
2.22 \text { ) }\end{array}$ & $\begin{array}{l}\text { Parallel-group randomized } \\
\text { trial ( } 3 \text { months) } \\
\text { DIAGNOdent }\end{array}$ & $\begin{array}{l}\text { Baseline: } 14.31 \pm 8.34 \\
\text { No increase over time }(\mathrm{p}=0.271) \\
\text { Baseline: } 14.14 \pm 10.32 \\
\text { Increased over time }(\mathrm{p}=0.002)\end{array}$ & $\begin{array}{l}\text { CPP-ACP had a slight } \\
\text { remineralization effect (lesions } \\
\text { not well defined, images suggest } \\
\text { some might be arrested at start) }\end{array}$ \\
\hline $\begin{array}{l}\text { Sim et al. } \\
\text { [2015] }\end{array}$ & $\begin{array}{l}0.4 \% \text { stannous fluoride gel } \\
\text { (Gel-Kam; Colgate) } 5 \text { min in } \\
\text { custom tray at night, } 10 \% \\
\text { CPP-ACP cream (GC) } 3 \times / \\
\text { day (at nighttime } 10 \text { min after } \\
\text { F gel), plus a } 0.32 \% \text { sodium } \\
\text { fluoride toothpaste (Colgate } \\
\text { Total) } \\
0.4 \% \text { stannous fluoride gel } \\
\text { (Gel-Kam; Colgate) } 5 \text { min in } \\
\text { custom tray at night, control } \\
\text { cream (GC) } 3 \times / \text { day } \\
\text { (nighttime } 10 \text { min after } \\
\text { F gel), plus a } 0.32 \% \text { sodium } \\
\text { fluoride toothpaste (Colgate } \\
\text { Total) }\end{array}$ & $\begin{array}{l}\mathrm{n}=24 \\
\text { Median age }=50 \text { years } \\
\text { (China; patients with } \\
\text { nasopharyngeal carcinoma } \\
\text { who received radiation } \\
\text { therapy; high risk due to } \\
\text { decreased salivary flow as } \\
\text { assessed after radiation) }\end{array}$ & $\begin{array}{l}\text { Double-blind, parallel-group } \\
\text { randomized trial ( } 3 \text { months) } \\
\text { ICDAS by } 4 \text { trained } \\
\text { examiners }\end{array}$ & $\begin{array}{l}\text { At } 3 \text { months: } 9 \text { subjects developed } 32 \\
\text { new lesions; } 93.4 \% \text { of lesions } \\
\text { remained stable; } 4.1 \% \text { of lesions } \\
\text { regressed; } 2.6 \% \text { of lesions progressed } \\
\text { There were no statistical significant } \\
\text { differences between the } 2 \text { groups } \\
\\
\text { At } 3 \text { months: } 8 \text { subjects developed } 59 \\
\text { new lesions; } 89.5 \% \text { of lesions } \\
\text { remained stable; } 5.6 \% \text { of lesions } \\
\text { regressed; } 5 \% \text { of lesions progressed }\end{array}$ & $\begin{array}{l}\text { Application of CPP-ACP did not } \\
\text { significantly reduce caries } \\
\text { progression in the first } 3 \text { months } \\
\text { after radiotherapy (sample size } \\
\text { small) }\end{array}$ \\
\hline $\begin{array}{l}\text { Plonka et al. } \\
\text { [2013] }\end{array}$ & $\begin{array}{l}10 \% \mathrm{CPP}-\mathrm{ACP} \text { paste } 1 \times / \text { day } \\
+2 \times / \text { day toothbrushing with } \\
0.304 \% \mathrm{~F} \text { toothpaste } \\
0.12 \% \text { chlorhexidine gel } 1 \times / \\
\text { day }+2 \times / \text { day toothbrushing } \\
\text { with } 0.304 \% \mathrm{~F} \text { toothpaste } \\
2 \times / \text { day toothbrushing with } \\
0.304 \% \mathrm{~F} \text { toothpaste }\end{array}$ & $\begin{array}{l}\mathrm{n}=622 \\
\text { Age: } 0-24 \text { months (focus } \\
\text { was ECC) }\end{array}$ & $\begin{array}{l}\text { Randomized controlled trial } \\
(24 \text { months }) \\
\text { Caries incidence }\end{array}$ & $\begin{array}{l}1 \%(2 / 163) \\
2 \%(4 / 180) \\
2 \%(3 / 188)\end{array}$ & $\begin{array}{l}\text { There is insufficient evidence to } \\
\text { justify daily use of CPP-ACP or } \\
\text { chlorhexidine gel to control ECC } \\
\text { (low caries incidence in all } \\
\text { groups) }\end{array}$ \\
\hline $\begin{array}{l}\text { Yazıcıoğlu and } \\
\text { Ulukapi [2013] }\end{array}$ & $\begin{array}{l}\text { Ozone (Healozone) applied } \\
\text { once for } 40 \mathrm{~s} \\
1.23 \% \text { APF gel (Sultan) } \\
\text { applied for } 1 \text { min } \\
\text { CPP-ACP (Tooth Mousse; } \\
\text { GC) applied locally plus given } \\
\text { for home use for 1 week; } \\
\text { Bonding agent (Clearfil } \\
\text { Protect Bond; Kuraray) } \\
\text { applied } \\
\text { Not treated }\end{array}$ & $\begin{array}{l}\mathrm{n}=42 \\
158 \text { noncavitated lesions } \\
\text { (Turkey; molars and } \\
\text { premolars with approximal } \\
\text { lesions were used, about } 25 \\
\text { lesions per group) }\end{array}$ & $\begin{array}{l}\text { Parallel-group trial (18 } \\
\text { months) } \\
\text { After tooth separation for } 2 \\
\text { days, approximal lesions } \\
\text { evaluated visually using } \\
\text { Ekstrand criteria, } \\
\text { radiographically, and with } \\
\text { DIAGNOdent }\end{array}$ & $\begin{array}{l}\begin{array}{l}\text { No progression visually }(\mathrm{p}<0.05) \text {, but } \\
\text { there was progression radiographically } \\
\text { and by DIAGNOdent }\end{array} \\
\text { No progression visually }(\mathrm{p}<0.05) \text {, but } \\
\text { there was progression radiographically } \\
\text { and by DIAGNOdent } \\
\text { No progression visually and } \\
\text { radiographically }(\mathrm{p}<0.05) \text {, but there } \\
\text { was progression by DIAGNOdent } \\
\text { No progression visually and by } \\
\text { DIAGNOdent ( } \mathrm{p}<0.05), \text { but there } \\
\text { was progression radiographically } \\
\text { Lesions progressed at } 18 \text { months } \\
\text { according to all methods }(\mathrm{p}<0.01)\end{array}$ & $\begin{array}{l}\text { Early-stage approximal lesions } \\
\text { remained stationary when } \\
\text { treated } \\
\text { Early lesions recovered with all } \\
\text { treatments (data unclear as } \\
\text { presented) }\end{array}$ \\
\hline
\end{tabular}


Table 1 (continued)

\begin{tabular}{|c|c|c|c|c|c|}
\hline Study & Test product & Subject characteristics & $\begin{array}{l}\text { Study design/ } \\
\text { outcome measure }\end{array}$ & Results & $\begin{array}{l}\text { Authors' conclusion } \\
\text { (reviewer comment) }\end{array}$ \\
\hline $\begin{array}{l}\text { Sitthisettapong } \\
\text { et al. [2012] }\end{array}$ & $\begin{array}{l}10 \% \text { CPP-ACP paste (Tooth } \\
\text { Mousse; GC) plus } 1,000 \mathrm{ppm} \\
\text { F toothpaste (paste applied in } \\
\text { school by trained teachers } \\
\text { after brushing) } \\
\text { Placebo paste (GC) plus } 1,000 \\
\text { ppm F toothpaste }\end{array}$ & $\begin{array}{l}\mathrm{n}=296 \\
\text { Preschool children: } 2.5-3.5 \\
\text { years old (Thailand; high } \\
\text { caries risk according to } \\
\text { caries risk tool and baseline } \\
\text { dmft around 4.3) }\end{array}$ & $\begin{array}{l}\text { Double-blind, randomized, } \\
\text { placebo-controlled clinical } \\
\text { trial ( } 1 \text { year) } \\
\text { ICDAS by } 1 \text { calibrated } \\
\text { examiner }\end{array}$ & $\begin{array}{l}\text { Baseline D5-6mfs: } 9.14+10.56 \\
1 \text { year D5-6mfs: } 13.37 \pm-13.8 \\
\text { Caries increment at } 1 \text { year: } 4.23 \pm 5.14 \\
\text { No significant difference amongst the } \\
2 \text { groups } \\
\text { Baseline D5-6mfs: } 9.38 \pm 12.91 \\
\text { 1-year D5-6mfs: } 13.50 \pm 15.91 \\
\text { Caries increment at } 1 \text { year: } 4.13 \pm 5.08\end{array}$ & $\begin{array}{l}\text { CPP-ACP use on school days in } \\
\text { addition to brushing with a } \\
\text { fluoridated toothpaste had no } \\
\text { significant added effect in } \\
\text { preventing caries }\end{array}$ \\
\hline $\begin{array}{l}\text { Bröchner } \\
\text { et al. [2011] } \\
\text { Listed in the } \\
\text { following } \\
\text { systematic } \\
\text { reviews: Chen } \\
\text { et al. [2013]; } \\
\text { Tellez et al. } \\
\text { [2013] }\end{array}$ & $\begin{array}{l}10 \% \text { CPP-ACP (Tooth } \\
\text { Mousse; GC) } \\
1 \times / \text { day in the evening }+ \\
\text { fluoride toothpaste (Colgate, } \\
1,100 \text { ppm F) in the morning }\end{array}$ & $\begin{array}{l}\mathrm{n}=60 \\
\text { Mean age: } 15.2 \text { years (range: } \\
\text { 13-18) } \\
\text { (Denmark; patients with } \\
\text { buccal white spot lesions } \\
\text { after removing brackets; } \\
\text { low level of fluoride in } \\
\text { water) }\end{array}$ & $\begin{array}{l}\text { Prospective, randomized, } \\
\text { single-blinded controlled } \\
\text { trial (no placebo) with } 2 \\
\text { parallel groups ( } 4 \text { weeks) } \\
\text { Clinical scores from } \\
\text { photographs, and QLF from } \\
\text { buccal surfaces } \\
\text { Data were analyzed by teeth, } \\
\text { not patient }\end{array}$ & $\begin{array}{l}\text { Baseline: prevalence white spot } \\
\text { lesions: } 84.6 \% \\
\text { Baseline } \triangle \mathrm{F}: 6.68 \pm 0.58 \\
\text { Baseline area: } 0.12 \pm 0.16 \\
4 \text { weeks: PCS (score 1): } 47.7 \% \\
4 \text { weeks } \Delta \mathrm{F}: 4.45 \pm 1.82 \\
4 \text { weeks area: } 0.05 \pm 0.09 \text { (decreased } \\
58 \% \text { ) } \\
4 \text { weeks prevalence of white spot } \\
\text { lesions: } 52.3 \% \\
\text { There were no statistically significant } \\
\text { differences between the groups } \\
\text { Baseline prevalence white spot lesions: } \\
85.1 \% \\
\text { Baseline } \Delta \mathrm{F}: 7.04 \pm 1.65 \\
\text { Baseline area: } 0.19 \pm 0.43 \\
4 \text { weeks: PCS (score } 1): 52.7 \% \\
4 \text { weeks } \Delta \mathrm{F}: 4.51 \pm 2.46 \\
4 \text { weeks area: } 0.14+0.31 \text { (decreased } \\
26 \% \text { ) } \\
4 \text { weeks prevalence of white spot } \\
\text { lesions: } 47.3 \%\end{array}$ & $\begin{array}{l}\text { A statistically significant } \\
\text { regression (around } 30-35 \% \text { ) of } \\
\text { white spot lesions was noted for } \\
\text { both groups compared to } \\
\text { baseline, with no difference } \\
\text { among the groups; thus, the } \\
\text { improvement was not superior } \\
\text { to regression with daily use of } \\
\text { fluoride toothpaste (according to } \\
\text { Chen et al. [2013]: high risk of } \\
\text { bias; according to Tellez et al. } \\
\text { [2013]: moderate risk of bias by } \\
\text { Cochrane, poor quality } \\
\text { according to ADA) }\end{array}$ \\
\hline $\begin{array}{l}\text { Altenburger et } \\
\text { al. [2010] } \\
\text { Listed in the } \\
\text { following } \\
\text { systematic } \\
\text { review: } \\
\text { Tellez et al. } \\
\text { [2013] }\end{array}$ & $\begin{array}{l}\text { CPP-ACP toothpaste } 1 \times / \text { day } \\
+1,450 \text { ppm F toothpaste } \\
1,450 \text { ppm F toothpaste }\end{array}$ & $\begin{array}{l}\mathrm{n}=32 \\
\text { Age: } 22-31 \text { years } \\
\text { (fissures with white spot } \\
\text { lesions on molar or } \\
\text { premolars, and } \\
\text { DIAGNOdent readings } \\
\text { 15-20) }\end{array}$ & $\begin{array}{l}\text { ( } 3 \text { weeks) } \\
\text { DIAGNOdent ( } 2 \text { calibrated } \\
\text { examiners) }\end{array}$ & $\begin{array}{l}\text { Baseline: } 16.66 \pm 1.27 \\
3 \text { weeks: } 10.96 \\
\text { No significant difference among the } 2 \\
\text { groups } \\
\text { Baseline: } 16.87 \pm 1.69 \\
3 \text { weeks: } 14.78\end{array}$ & $\begin{array}{l}\text { No difference among the } 2 \\
\text { treatments } \\
\text { (according to review: low risk of } \\
\text { bias by Cochrane, and poor } \\
\text { quality by ADA) }\end{array}$ \\
\hline
\end{tabular}




\begin{tabular}{|c|c|c|c|c|c|}
\hline Study & Test product & Subject characteristics & $\begin{array}{l}\text { Study design/ } \\
\text { outcome measure }\end{array}$ & Results & $\begin{array}{l}\text { Authors' conclusion } \\
\text { (reviewer comment) }\end{array}$ \\
\hline $\begin{array}{l}\text { Bailey et al. } \\
\text { [2009] } \\
\text { Listed in the } \\
\text { following } \\
\text { systematic } \\
\text { reviews: Tellez } \\
\text { et al. [2013]; } \\
\text { Chen et al. } \\
\text { [2013] }\end{array}$ & $\begin{array}{l}\text { 10\% CPP-ACP (Tooth } \\
\text { Mousse; GC) } 2 \times / \text { day after F } \\
\text { toothpaste }+\mathrm{F} \text { toothpaste } \\
(1,000 \mathrm{ppm} \mathrm{F} \text { as NaF) daily + } \\
\text { supervised NaF mouthrinse } \\
(900 \mathrm{ppm} \mathrm{F}) \text { during } \\
\text { assessment visits } \\
\text { Placebo cream } 2 \times / \text { day after F } \\
\text { toothpaste }+\mathrm{F} \text { toothpaste } \\
(1,000 \mathrm{ppm} \text { F) daily + } \\
\text { supervised NaF mouthrinse } \\
(900 \text { ppm F) during } \\
\text { assessment visits }\end{array}$ & $\begin{array}{l}\mathrm{n}=45 \\
\text { Adolescents: } 12-18 \text { years } \\
\text { old (Australia; patients had } \\
\text { at least } 2 \text { white spot lesions, } \\
\text { the majority assessed as } \\
\text { ICDAS } 2-3 \text {, after removal } \\
\text { of orthodontic brackets) }\end{array}$ & $\begin{array}{l}\text { Randomized, double-blind, } \\
\text { controlled clinical trial with } \\
2 \text { parallel groups (12 weeks) } \\
\text { Visual (ICDAS) for severity } \\
\text { and activity (regression } \\
\text { considered if lesion less } \\
\text { severe or less active) by } 3 \\
\text { calibrated examiners (this } \\
\text { was a secondary outcome of } \\
\text { trial which included as } \\
\text { primary outcomes } \\
\text { photographs and QLF) }\end{array}$ & $\begin{array}{l}\text { Baseline to } 12 \text { weeks - lesion } \\
\text { transition score changes: OR: } 1.67 \\
\text { ( } 95 \% \text { CI: } 0.81-3.45) \text {, not significant } \\
\text { between groups } \\
\text { Only significant for lesions } 2-3 \text {; OR: } \\
2.33(1.06-5.14): 31 \% \text { more regressed } \\
\text { in the test group compared to placebo }\end{array}$ & $\begin{array}{l}\text { Significantly more white spot } \\
\text { lesions regressed with the CPP- } \\
\text { ACP cream compared to placebo } \\
\text { over } 12 \text { months (according to } \\
\text { Tellez et al. [2013]: low risk of } \\
\text { bias by Cochrane, poor quality } \\
\text { by ADA; } \\
\text { according to Chen et al. [2013]: } \\
\text { high risk of bias; primary QLF } \\
\text { outcome data not reported as } \\
\text { authors stated it was unreliable } \\
\text { due to gingival location of } \\
\text { lesions) }\end{array}$ \\
\hline $\begin{array}{l}\text { Andersson et } \\
\text { al. [2007] } \\
\text { Listed in the } \\
\text { following } \\
\text { systematic } \\
\text { reviews: } \\
\text { Azarpazhooh } \\
\text { and Limeback } \\
\text { [2008]; Chen et } \\
\text { al. [2013]; } \\
\text { Tellez et al. } \\
\text { [2013] }\end{array}$ & $\begin{array}{l}\text { CPP-ACP cream (Topacal } \\
\text { C- } 5 \text {; not Recaldent but } \\
\text { Phoscal) for } 3 \text { months }+ \text { F } \\
\text { toothpaste }(1,000-1,100 \mathrm{ppm} \\
\text { F) for next } 3 \text { months }\end{array}$ & $\begin{array}{l}\mathrm{n}=26 \\
\text { Adolescents, mean age: } 14.6 \\
\text { years, range: } 12-16 \\
\text { (Sweden; } 60 \text { teeth, } 152 \\
\text { visible - without air-drying } \\
\text { - white spot lesions after } \\
\text { debonding brackets; } \\
\text { patients reported use of F } \\
\text { toothpaste } 2 \times / \text { daily, lived in } \\
\text { an area with low F in the } \\
\text { water, and had developed } \\
\text { lesions during orthodontics } \\
\text { despite daily use of } 0.05 \% \\
\text { NaF rinse) }\end{array}$ & $\begin{array}{l}\text { Randomized clinical trial } \\
\text { with } 2 \text { parallel groups, blind } \\
\text { assessment (12 months) } \\
\text { Clinical (visual; scores } 0-4) \\
\text { and DIAGNOdent by } 1 \\
\text { calibrated examiner } \\
\text { Data were analyzed by teeth, } \\
\text { not patient }\end{array}$ & $\begin{array}{l}\text { DIAGNOdent: baseline: } 7.4 \pm 10.2 \\
12 \text { months: } 4.4 \pm 5.2 \text { (significantly less } \\
\text { than baseline) } \\
\text { Difference of } 0-12 \text { months between } \\
\text { sites that were scored } 0-1 \text { at end: } 63 \% \\
\text { No significant difference with control } \\
\text { for DIAGNOdent readings, but a } \\
\text { significantly greater visual } \\
\text { improvement in the remineralizing } \\
\text { group compared to control } \\
\text { Baseline: } 9.4 \pm 9.5 \\
12 \text { months: } 6.4 \pm 7.5 \text { (significantly less } \\
\text { than baseline) } \\
\text { Difference of } 0-12 \text { months between } \\
\text { sites that were scored } 0-1 \text { at end: } 25 \%\end{array}$ & $\begin{array}{l}\text { Both treatments } \\
\text { reversed white spot lesions after } \\
\text { orthodontic treatment; better } \\
\text { visual outcome for CPP-ACP } \\
\text { group (according to } \\
\text { Azarpazhooh and Limeback } \\
\text { [2008]: single-blinded RCT, } \\
\text { small sample, no power } \\
\text { calculation; subjective scoring; } \\
\text { no significant difference with } \\
\text { laser fluorescence; } \\
\text { according to Chen et al. [2013]: } \\
\text { high risk of bias; according to } \\
\text { Tellez et al. [2013]: moderate } \\
\text { risk of bias by Cochrane, poor } \\
\text { quality by ADA) }\end{array}$ \\
\hline \multicolumn{6}{|c|}{ CPP-ACFP studies (MI Paste Plus) } \\
\hline $\begin{array}{l}\text { Huang et al. } \\
\text { [2013] }\end{array}$ & $\begin{array}{l}\text { PreviDent fluoride varnish } \\
\text { (Colgate), } 1 \text { application }\end{array}$ & $\begin{array}{l}\mathrm{n}=115 \\
\text { Age: } 12-20 \text { years (the } \\
\text { patients had their } \\
\text { orthodontic appliances } \\
\text { removed within the past } 2 \\
\text { months and had at least } 1 \\
\text { white spot lesion affecting } \\
\text { their maxillary incisors) }\end{array}$ & $\begin{array}{l}\text { Parallel-group randomized } \\
\text { trial ( } 8 \text { weeks) } \\
\text { Photographs assessed by } \\
\text { several groups }\end{array}$ & $\begin{array}{l}\text { Mean improvement assessed by: } \\
\text { (1) The professional panel = } 21 \% \\
\text { (2) The lay panel = } 29 \% \\
\text { (3) Objective improvement }=16 \% \\
\text { (4) Self-assessment = } 37 \% \\
\text { No significant differences between } \\
\text { subjects in the active arms compared } \\
\text { to the control arm } \\
\text { Mean improvement assessed by: } \\
\text { (1) The professional panel = 29\% } \\
\text { (2) The lay panel = 31\% } \\
\text { (3) Objective improvement = } 25 \% \\
\text { (4) Self-assessment = 37\% } \\
\text { Mean improvement assessed by: } \\
\text { (1) The professional panel = } 27 \% \\
\text { (2) The lay panel }=25 \% \\
\text { (3) Objective improvement }=17 \% \\
\text { (4) Self-assessment }=37 \%\end{array}$ & $\begin{array}{l}\text { MI Paste Plus and PreviDent } \\
\text { fluoride varnish do not appear } \\
\text { to be more effective than normal } \\
\text { home care for improving the } \\
\text { appearance of white spot lesions } \\
\text { over an 8-week period }\end{array}$ \\
\hline
\end{tabular}


Table 1 (continued)

\begin{tabular}{|c|c|c|c|c|c|}
\hline Study & Test product & Subject characteristics & $\begin{array}{l}\text { Study design/ } \\
\text { outcome measure }\end{array}$ & Results & $\begin{array}{l}\text { Authors' conclusion } \\
\text { (reviewer comment) }\end{array}$ \\
\hline $\begin{array}{l}\text { Robertson et } \\
\text { al. [2011] }\end{array}$ & $\begin{array}{l}\text { Place Paste (Tom's of Maine), } \\
\text { used in a tray } 3-5 \mathrm{~min} / \text { day at } \\
\text { night after brushing }\end{array}$ & $\begin{array}{l}\mathrm{n}=60 \\
\text { Age: } 12 \text { years and older } \\
\text { (USA; patients undergoing } \\
\text { orthodontic treatment) }\end{array}$ & $\begin{array}{l}\text { Prospective, double-blind, } \\
\text { randomized trial ( } 3 \text { months) } \\
\text { Visual through photographs } \\
\text { using ICDAS by } 3 \text { trained } \\
\text { examiners (enamel } \\
\text { decalcification index, EDI: } \\
\text { number of white spot lesions } \\
\text { per surface) }\end{array}$ & $\begin{array}{l}\text { EDI baseline: } 271 \\
\text { EDI } 3 \text { months: } 126 \text { (reduced } 53.5 \%) \\
\text { Differences between groups were } \\
\text { statistically significant } \\
\text { ICDAS (change in sum of scores): } \\
44.8 \% \text { reduction over } 3 \text { months (all } \\
\text { ICDAS results were not significant) } \\
\text { EDI baseline: } 135 \\
\text { EDI } 3 \text { months: } 258 \text { (increased 91.1\%) } \\
\text { ICDAS (change in sum of scores): } \\
43.1 \% \text { increase over } 3 \text { months }\end{array}$ & $\begin{array}{l}\text { MI Paste Plus helped prevent the } \\
\text { development of new white spot } \\
\text { lesions during orthodontic } \\
\text { treatment and decreased the } \\
\text { number of lesions already } \\
\text { present (many of the white spots } \\
\text { measured were on the incisal } \\
\text { edge, so unlikely caries lesions } \\
\text { only included) }\end{array}$ \\
\hline $\begin{array}{l}\text { Beerens et al. } \\
\text { [2010] } \\
\text { Listed in the } \\
\text { following } \\
\text { systematic } \\
\text { reviews: Tellez } \\
\text { et al. [2013]; } \\
\text { Chen et al. } \\
\text { [2013] }\end{array}$ & $\begin{array}{l}\text { CPP-ACP }+ \text { NaF }(0.2 \%, 900 \\
\text { ppm; MI Paste Plus) } 1 \times / \text { day } \\
\text { before bedtime to supplement } \\
\text { normal oral hygiene } \\
\text { (recommended } 2 \times / \text { day } \\
\text { fluoridated toothpaste) } \\
\text { F-free paste }+ \text { calcium } \\
\text { (Ultradent) } 1 \times / \text { day before } \\
\text { bedtime to supplement } \\
\text { normal oral hygiene } \\
\text { (recommended } 2 \times / \text { day } \\
\text { fluoridated toothpaste) }\end{array}$ & $\begin{array}{l}\mathrm{n}=65 \\
\text { Mean age: } 15.5 \text { years, range: } \\
12-19 \text { (The Netherlands; } \\
\text { no exposure to fluoridated } \\
\text { water, patients had buccal } \\
\text { white spot lesions seen } \\
\text { without air drying prior to } \\
\text { removal of brackets) }\end{array}$ & $\begin{array}{l}\text { Double-blind, prospective } \\
\text { randomized clinical trial (12 } \\
\text { weeks) } \\
\text { QLF + visual (did not use } \\
\text { ICDAS as lesions were } \\
\text { primarily 2-3, so difficult to } \\
\text { show change) }\end{array}$ & $\begin{array}{l}\text { Baseline } \Delta \mathrm{F}: 8.45 \pm 1.17 \\
\text { Baseline area: } 5.07 \pm 5.69 \\
12 \text { weeks } \Delta \mathrm{F}: 7.52 \pm 1.78 \\
12 \text { weeks area: } 0.05 \pm 6.98 \\
\text { Groups were not significantly } \\
\text { different (compliance was moderate) } \\
\text { Baseline } \Delta \mathrm{F}: 9.10 \pm 1.75 \\
\text { Baseline area: } 7.29 \pm 7.91 \\
12 \text { weeks } \Delta \mathrm{F}: 7.96 \pm 2.76 \\
12 \text { weeks area: } 7.17 \pm 7.76\end{array}$ & $\begin{array}{l}\text { A significant decrease in } \\
\text { fluorescence loss was found with } \\
\text { respect to baseline for both } \\
\text { groups, with no difference } \\
\text { among them; the area of the } \\
\text { lesions did not change over time } \\
\text { or between groups; thus, no } \\
\text { clinical advantage was noted for } \\
\text { use of CPP-ACFP paste } \\
\text { supplementary to oral hygiene } \\
\text { over } 12 \text { weeks - short time span } \\
\text { to see changes (according to } \\
\text { Tellez et al. [2013]: moderate } \\
\text { risk of bias by Cochrane and } \\
\text { poor quality by ADA; } \\
\text { according to Chen et al. [2013]: } \\
\text { high risk of bias) }\end{array}$ \\
\hline
\end{tabular}

Casein derivatives coupled with calcium phosphate

Thomson

[2002]

Listed in the

following

systematic

review:

Azarpazhooh

and Limeback

[2008]

Self-administered topical

casein derivatives coupled

with calcium phosphate (CD-

CP; Gencal Dental

Conditioner) mouthrinse $3 \times 1$

day

Self-administered topical

$0.05 \% \mathrm{NaF}$ mouthrinse $3 \times$

day $\mathrm{n}=138$

Adults aged $53 \pm 14$ year

(New Zealand; patients with

salivary gland dysfunction:

$0.1 \mathrm{ml} / \mathrm{min}$ or less resting

saliva flow - radiotherapy

or Sjögren's syndrome

and dry mouth)
Randomized control clinical trial with 2 parallel group (12 months)

Bitewing radiographs

(coronal caries increment)
Coronal increment: $34.4 \%$

The results showed no difference between the $\mathrm{NaF}$ mouthrinse and the $\mathrm{CD}-\mathrm{CP}$ mouthrinse with regard to caries-preventive efficacy

Coronal increment: $27 \%$
No difference between a NaF mouthrinse and a CD-CP mouthrinse in their cariespreventive efficacy; CD-CP may hold promise for caries prevention in dry mouth syndrome - sample size was smaller than calculated due to difficulties recruiting according to systematic review: RCT - double-blinding unclear; small sample for $90 \%$ power type II error; no control over care outside study) 


\begin{tabular}{|c|c|c|c|c|c|}
\hline Study & Test product & Subject characteristics & $\begin{array}{l}\text { Study design/ } \\
\text { outcome measure }\end{array}$ & Results & $\begin{array}{l}\text { Authors' conclusion } \\
\text { (reviewer comment) }\end{array}$ \\
\hline \multirow[t]{2}{*}{$\begin{array}{l}\text { ACP studies } \\
\text { Rao et al. } \\
{[2009]}\end{array}$} & $\begin{array}{l}\text { 2\% w/w CPP toothpaste } \\
\text { (Institute of Medical Sciences, } \\
\text { Bangalore), supervised } \\
\text { brushing } 2 \mathrm{x} / \text { day at school; } \\
\text { children were asked to refrain } \\
\text { from other oral hygiene } \\
\text { practices and not to use any } \\
\text { other substances or } \\
\text { dentifrices to clean their teeth } \\
\text { during the study (all groups) }\end{array}$ & $\begin{array}{l}\mathrm{n}=150 \\
\text { Schoolchildren: } 12-15 \\
\text { years old (India; high caries } \\
\text { risk measured by DMFT }>2 ; \\
\text { low socioeconomic status) }\end{array}$ & $\begin{array}{l}\text { Randomized, double- } \\
\text { blinded, controlled clinical } \\
\text { trial ( } 24 \text { months) } \\
\text { DMFS using WHO criteria } \\
\text { by } 1 \text { calibrated examiner } \\
\text { (caries increment) }\end{array}$ & $\begin{array}{l}\triangle \mathrm{DS} \text { at } 24 \text { months: } 0.22 \pm 0.44 \\
72.3 \% \text { remained caries free } \\
\text { A significant reduction in caries } \\
\text { increment was observed among } \\
\text { students using CPP or MFP } \\
\text { toothpaste (but no difference amongst } \\
\text { them), compared to the group using } \\
\text { the placebo toothpaste }\end{array}$ & \multirow[t]{2}{*}{$\begin{array}{l}\text { CPP can be effectively } \\
\text { incorporated into calcium } \\
\text { carbonate-based toothpaste, and } \\
\text { toothpaste containing CPP is } \\
\text { effective in preventing caries; } \\
\text { toothpaste containing } 2 \% \text { CPP } \\
\text { seemed to have an efficacy } \\
\text { similar to paste containing } 1,190 \\
\text { ppm MFP in the prevention of } \\
\text { caries (ethical concern regarding } \\
\text { use of placebo for } 2 \text { years) }\end{array}$} \\
\hline & $\begin{array}{l}\text { Placebo toothpaste without } \\
\text { CPP or fluoride (Institute of } \\
\text { Medical Sciences, Bangalore), } \\
\text { supervised brushing } 2 \times / \text { day } \\
\text { at school }\end{array}$ & & & $\begin{array}{l}\mathrm{DS} \text { at } 24 \text { months: } 0.62+0.92 \\
31.1 \% \text { remained caries free }\end{array}$ & \\
\hline $\begin{array}{l}\text { Papas et al. } \\
\text { [1999] }\end{array}$ & $\begin{array}{l}\text { ACPF toothpaste (dual-phase } \\
\text { remineralizing toothpaste, } \\
\text { Enamelon, with } 1,100 \mathrm{ppm} \mathrm{F} \\
\text { and soluble calcium and } \\
\text { phosphate ions, from separate } \\
\text { phases) + caries restored at } \\
\text { baseline and F varnish } \\
\text { (Duraphat) applied + daily } \\
0.05 \% \text { NaF alcohol-free rinse } \\
+ \text { pilocarpine } 5 \text { mg q.i.d. } \\
\text { NaF toothpaste (1,100 ppm } \\
\text { F) + caries restored at } \\
\text { baseline and F varnish } \\
\text { (Duraphat) applied + daily } \\
0.05 \% \text { NaF alcohol-free rinse } \\
+ \text { pilocarpine } 5 \text { mg q.i.d. }\end{array}$ & $\begin{array}{l}\mathrm{n}=57(44 \text { finished })(\text { Head } \\
\text { and neck post-radiation } \\
\text { patients; saliva flow less } \\
\text { than } 0.2 \mathrm{ml} / \text { min resting) }\end{array}$ & $\begin{array}{l}\text { Double-blind randomized } \\
\text { clinical trial } \\
\text { (10-12 months) } \\
\text { Coronal and root caries by } 1 \\
\text { calibrated examiner using } \\
\text { the Pitts Diagnostic Criteria }\end{array}$ & $\begin{array}{l}\text { Average net increment per year for } \\
\text { root caries per subject was } 0.04 \pm 0.052 \\
\text { No significant difference in coronal } \\
\text { caries increment, but significantly less } \\
\text { root caries }\end{array}$ & $\begin{array}{l}\text { The results indicate that the } \\
\text { remineralizing toothpaste } \\
\text { provides a significant benefit in } \\
\text { preventing and remineralizing } \\
\text { root caries in high-risk patients } \\
\text { (long-term studies are needed as } \\
\text { there is the concern on } \\
\text { promotion of calculus with this } \\
\text { technology) }\end{array}$ \\
\hline $\begin{array}{l}\mathrm{CaF}_{2} \\
\text { Ferreira et al. } \\
\text { [2009] }\end{array}$ & $\begin{array}{l}5 \% \text { NaF Varnish (Duraphat; } \\
\text { Colgate) weekly (used } 1,500 \\
\text { ppm F Colgate toothpaste at } \\
\text { home) } \\
5 \% \mathrm{NaF}+6 \% \mathrm{CaF}_{2} \\
\text { (Duofluorid) varnish weekly } \\
\text { (used } 1,500 \text { ppm F Colgate } \\
\text { toothpaste at home) }\end{array}$ & $\begin{array}{l}\mathrm{n}=15 \\
\text { Age: } 7-12 \text { years (Brazil; } \\
\text { effect on anterior active } \\
\text { white spot lesions; no } \mathrm{F} \text { in } \\
\text { water) }\end{array}$ & $\begin{array}{l}\text { Randomized clinical trial ( } 4 \\
\text { weeks) } \\
\text { Lesion size measured with } \\
\text { periodontal probe, and } \\
\text { activity ( } 1 \text { calibrated } \\
\text { examiner) }\end{array}$ & $\begin{array}{l}\text { Reduction in mm: } 1.19 \\
\text { Inactive lesions at } 4 \text { weeks: } 15 \\
\text { Similar reductions were observed in } \\
\text { both varnish groups; no difference } \\
\text { was observed between the groups } \\
\text { regarding activity scores } \\
\text { Reduction in mm: } 1.29 \\
\text { Inactive lesions at } 4 \text { weeks: } 21\end{array}$ & $\begin{array}{l}\text { The two varnish formulations } \\
\text { tested produced similar clinical } \\
\text { effects, indicating the reduction } \\
\text { and the control of carious } \\
\text { activity in most lesions (outcome } \\
\text { not common) }\end{array}$ \\
\hline
\end{tabular}


Table 1 (continued)

\begin{tabular}{|c|c|c|c|c|c|}
\hline Study & Test product & Subject characteristics & $\begin{array}{l}\text { Study design/ } \\
\text { outcome measure }\end{array}$ & Results & $\begin{array}{l}\text { Authors' conclusion } \\
\text { (reviewer comment) }\end{array}$ \\
\hline \multicolumn{6}{|c|}{ Dicalcium phosphate dehydrate } \\
\hline $\begin{array}{l}\text { Boneta et al. } \\
\text { [2001] }\end{array}$ & $\begin{array}{l}\text { Dentifrice system in a dual- } \\
\text { chambered tube ( } 1 \text { chamber: } \\
\text { sodium fluoride in a silica } \\
\text { base and the other chamber } \\
\text { contained dicalcium } \\
\text { phosphate dehydrate) } \\
\text { delivering } 0.243 \% \text { sodium } \\
\text { fluoride } 2 \times / \text { day }\end{array}$ & $\begin{array}{l}\mathrm{n}=2,506 \\
\text { Children } 5-17 \text { years old } \\
\text { (US and Puerto Rico) }\end{array}$ & $\begin{array}{l}\text { Double-blind, parallel-group } \\
\text { randomized trial ( } 2 \text { years }) \\
\text { Visual, by } 1 \text { calibrated } \\
\text { examiner }\end{array}$ & $\begin{array}{l}\text { DMFS at baseline: } 2.29 \\
\text { Caries increment at } 2 \text { years: } 1.25 \\
\text { At } 2 \text { years the dentifrices were } \\
\text { significantly different, with the test } \\
\text { dentifrice having a } 14.38 \% \text { reduction } \\
\text { in caries increment compared to the } \\
\text { control }\end{array}$ & \multirow[t]{2}{*}{$\begin{array}{l}\text { The test dentifrice provided a } \\
\text { superior level of anticaries } \\
\text { efficacy than the control }\end{array}$} \\
\hline & $\begin{array}{l}\text { Dentifrice containing } 0.243 \% \\
\text { sodium fluoride in a silica } \\
\text { base (positive control } \\
\text { dentifrice) } 2 \times / \text { day }\end{array}$ & & & $\begin{array}{l}\text { DMFS at baseline: } 2.47 \\
\text { Caries increment at } 2 \text { years: } 1.46\end{array}$ & \\
\hline \multirow[t]{2}{*}{$\begin{array}{l}\text { Silva et al. } \\
\text { [2001] }\end{array}$} & $\begin{array}{l}\text { Dentifrice system in a dual- } \\
\text { chambered tube, wherein } 1 \\
\text { chamber contained sodium } \\
\text { fluoride in a silica base and } \\
\text { the other chamber contained } \\
\text { dicalcium phosphate } \\
\text { dehydrate (test dentifrice } \\
\text { delivering } 0.243 \% \text { sodium } \\
\text { fluoride) } 2 \times / \text { day }\end{array}$ & $\begin{array}{l}\mathrm{n}=2,432 \\
\text { Children } 6-10 \text { years old } \\
\text { (Brazil; low level of } \mathrm{F} \text { in } \\
\text { water) }\end{array}$ & $\begin{array}{l}\text { Double-blind, parallel-group } \\
\text { randomized trial ( } 2 \text { years) } \\
\text { Visual, by } 1 \text { calibrated } \\
\text { examiner }\end{array}$ & $\begin{array}{l}\text { DMFS at baseline: } 3.84 \\
\text { Caries increment at } 2 \text { years: } 4.30 \\
\text { At } 2 \text { years the dentifrices were } \\
\text { significantly different, with the test } \\
\text { dentifrice having a } 10.97 \% \text { reduction } \\
\text { in caries increment compared to the } \\
\text { control }\end{array}$ & \multirow[t]{2}{*}{$\begin{array}{l}\text { The test dentifrice provided a } \\
\text { superior level of anticaries } \\
\text { efficacy than the control }\end{array}$} \\
\hline & $\begin{array}{l}\text { Dentifrice containing } 0.243 \% \\
\text { sodium fluoride in a silica } \\
\text { base (positive control } \\
\text { dentifrice) } 2 \times / \text { day }\end{array}$ & & & $\begin{array}{l}\text { DMFS at baseline: } 4.06 \\
\text { Caries increment at } 2 \text { years: } 4.83\end{array}$ & \\
\hline
\end{tabular}


Table 2. Summary statistics for QLF studies conducted on noncavitated enamel lesions to assess the efficacy of toothpastes containing $1.5 \%$ arginine, 1,450 ppm $\mathrm{F}$ in an insoluble calcium base

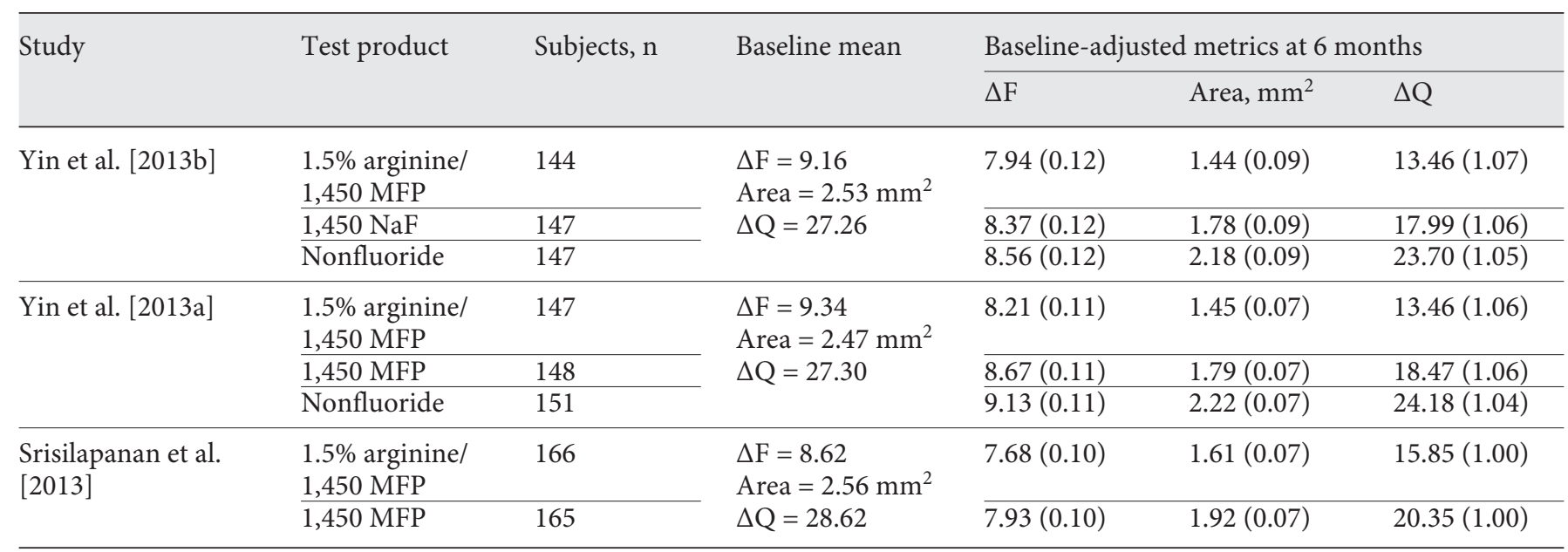

Values in parentheses are standard errors.

Table 3. Summary statistics for root caries studies conducted to assess the efficacy of toothpastes containing $1 / 5 \%$ arginine, 1,450 ppm $\mathrm{F}$ in an insoluble calcium base

\begin{tabular}{|c|c|c|c|c|c|c|c|c|}
\hline \multirow[t]{2}{*}{ Author } & \multirow[t]{2}{*}{ Test product } & \multirow[t]{2}{*}{ Subjects, $\mathrm{n}$} & \multirow{2}{*}{\multicolumn{2}{|c|}{$\begin{array}{l}\text { Subject baseline lesion } \\
\text { status }\end{array}$}} & \multicolumn{4}{|c|}{ 6-month lesion status, n (\%) } \\
\hline & & & & & hard & leathery & soft & $\begin{array}{l}\text { filled or } \\
\text { extracted }\end{array}$ \\
\hline \multirow{6}{*}{$\begin{array}{l}\text { Hu et al. } \\
{[2013]}\end{array}$} & $1.5 \%$ arginine/ & 141 & Leathery & $\mathrm{n}=84$ & $55(65.5)$ & $28(33.3)$ & $1(1.2)$ & \\
\hline & 1,450 MFP & & Soft & $\mathrm{n}=57$ & $8(14.0)$ & $24(42.1)$ & $25(43.9)$ & \\
\hline & $1,450 \mathrm{NaF}$ & 134 & Leathery & $\mathrm{n}=79$ & $47(59.5)$ & $20(25.3)$ & $12(15.2)$ & $91(70.5)$ \\
\hline & & & Soft & $\mathrm{n}=55$ & $0(0.0)$ & $28(50.9)$ & $27(49.1)$ & \\
\hline & Nonfluoride & 137 & Leathery & $\mathrm{n}=80$ & $20(25.0)$ & $35(43.8)$ & $25(31.3)$ & $72(58.1)$ \\
\hline & & & Soft & $\mathrm{n}=57$ & $0(0.0)$ & $17(29.8)$ & $40(70.2)$ & \\
\hline \multirow[t]{2}{*}{$\begin{array}{l}\text { Souza et al. } \\
\text { [2013] }\end{array}$} & $\begin{array}{l}1.5 \% \text { arginine/ } \\
1,450 \mathrm{MFP}\end{array}$ & 129 & Leathery & $\mathrm{n}=129$ & $91(70.5)$ & $34(26.4)$ & $0(0.0)$ & $4(3.1)$ \\
\hline & $1,450 \mathrm{MFP}$ & 124 & Leathery & $\mathrm{n}=124$ & $72(58.1)$ & $47(37.9)$ & $2(1.6)$ & $3(2.4)$ \\
\hline
\end{tabular}

maining studies focusing on the addition of microbial-related strategies to a fluoride-containing vehicle (2 xylitol studies, plus 1 study using a probiotic milk) favored the combination as a booster to fluoride-only control arms.

\section{Discussion}

Fluoride-free caries management strategies with a similar or enhanced effect compared to fluoride would be an attractive alternative for remineralization, especially for patient groups reluctant to using fluoride products or for young children at risk of dental fluorosis. Furthermore, strategies that would enhance or boost the anticaries properties of fluoride would be of interest, especially for high-caries-risk patients such as those with decreased salivary flow, as fluoride treatments for promoting remineralization are limited by the lack of available calcium and phosphate ions in sufficient amounts, and thus less aggressive fluoride therapy might be possible if calcium and phosphate ions could be provided [Hay and Thomson, 2002]. 


\section{Calcium-Based Strategies}

Remineralization strategies, designed to boost fluoride or serve as an alternative to fluoride, are meant to increase the concentration of calcium and phosphate delivered to caries lesions and/or increase their concentrations in plaque and saliva. They are incorporated into a variety of delivery vehicles including toothpastes, creams, mouthrinses, varnishes and chewing gums/mints in different parts of the world. These strategies include CPP-ACP, ACP, bioactive glass containing calcium sodium phosphosilicate (Novamin), tricalcium phosphate, nanohydroxyapatite, dicalcium phosphate dehydrate, etc. [Amaechi and van Loveren, 2013]. Since plaque fluid is already supersaturated at a neutral $\mathrm{pH}$ with respect to calcium phosphate in the tooth, the benefit of adding extra calcium to enhance remineralization has been questioned. However, at a low $\mathrm{pH}$, the controlled release of calcium may reduce undersaturation with respect to the tooth, and may enhance fluoride 'reservoirs' at the tooth surface and fluoride-induced remineralization. Multiple challenges are associated with determining the effectiveness of remineralization strategies, including the need to diffuse through a biofilm, rapidly precipitate and stabilize into caries lesions, and provide a benefit on top of that of fluoride and saliva [Zero, 2009]. Newer formulations include fluoride incorporated into the product, resulting in CPP-ACFP or ACFP.

Casein Derivatives Coupled with Calcium

(CPP-ACP, CPP-ACFP, CD-CP)

The proposed mechanism of action for CPP-ACP (Tooth Mousse or MI Paste) and CPP-ACFP (Tooth Mousse Plus or MI Paste Plus) in promoting remineralization is that it can enter lesion porosities and diffuse following concentration gradients to the body of the lesion. There, calcium and phosphate ions are released and aid in remineralization. In the presence of fluoride this forms fluorapatite or fluorhydroxyapatite. In addition, the breakdown of the CPP can help rise the $\mathrm{pH}$ (buffer) through the production of ammonia, plus it may inhibit bacterial adhesion to the tooth, thus delaying biofilm formation [Cochrane et al., 2010]. Thirteen trials were included when reviewing casein and calcium phosphate combinations - 9 trials evaluated CPP-ACP, 3 trials evaluated CPP-ACFP, and 1 trial evaluated CD-CP [Hay and Thomson, 2002]. Sample sizes of the trials ranged from 24 to 622 , and were conducted between 3 weeks and 24 months. Most studies used the test product in addition to some form of fluoride exposure (e.g. fluoride toothpaste use twice a day). Eleven studies evaluated permanent den-

Fluoride Boosters and/or Alternatives tition (6/11 used some form of a light fluorescence technology to evaluate changes given the short term of the studies, 2/11 evaluated high-risk patients with dry mouth, and $7 / 11$ were done using white spots developed during orthodontic treatment), while 2 studies evaluated primary dentition (infants and preschool children assessed visually). The 2 studies in young children with CPP-ACP showed no significant added effect of CPP-ACP [Sitthisettapong et al., 2012; Plonka et al., 2013]. Two studies were done using patients with hyposalivation (and thus high risk for caries), and none of these favored the calcium test product [Hay and Thomson, 2002; Sim et al., 2015]. Out of the 7 studies done using white spot lesions developed around orthodontic brackets, 3 favored the calcium test agents CPP-ACP [Andersson et al., 2007; Bailey et al., 2009] and CPP-ACFP [Robertson et al., 2011]. Out of the 9 studies testing CPP-ACP, only 3 studies favored the test product (in 1 of them only when used in more advanced white spot lesions-ICDAS 2-3 [Bailey et al., 2009], and 1 of them found significant improvements only when measured visually and not with DIAGNOdent [Andersson et al., 2007]). The single study testing CD-CP did not favor the test agent [Hay and Thomson, 2002]. Of the 3 studies testing CPP-ACFP (MI Paste Plus), only 1 study favored the test product [Robertson et al., 2011].

Several reviews have been written about the use of calcium-based strategies for caries control, with different conclusions depending on the data reviewed. In some instances conclusions have been very promising even when clinical data available have been scarce. For example, Reynolds [2008] concluded that calcium-based remineralization strategies show promise as supplemental treatments to fluoride for noninvasive management of caries lesions, even when the review did not include any randomized controlled clinical data for Novamin, and included only 1 small clinical study using ACP plus a fluoride toothpaste in a group of high-risk head and neck radiation patients [Papas et al., 1999] and 1 randomized clinical trial using ACP-CPP in a gum (vehicle excluded in this review). That same year a systematic review of 12 clinical studies was published, concluding that 'the quantity and quality of evidence [up to that point] are insufficient to make conclusions about long-term effectiveness of casein derivatives, specifically CPP-ACP' [Azarpazhooh and Limeback, 2008]. The authors raised the concern that the majority of the studies were conducted by the same group of authors who held the CPP-ACP patent, and that the majority of clinical studies were done using an in situ design, which is considered a surrogate 
model for caries with unclear associations with clinical trials [Bader, 2010]. In addition, the authors noted discrepancies in study findings depending on how the outcome was measured (visually vs. using laser fluorescence). A year later, another systematic review by Yengopal and Mickenautsch [2009] of 5 in situ short-term studies (7-21 days; all excluded in the current study due to their design) concluded that the data supported a short-term remineralization effect of CPP-ACP. As stated by Bader [2010], this is an example of how 2 reviews [Azarpazhooh and Limeback, 2008; Yengopal and Mickenautsch, 2009] of almost the same literature can came to completely different conclusions depending on which studies they accept, and how they consider the quality of the evidence in reaching their conclusions. In 2013, a systematic review of 7 randomized controlled trials of remineralizing agents for the treatment of postorthodontic white spot lesions concluded there was a lack of reliable evidence to support the effectiveness of calcium-based strategies for remineralization [Chen et al., 2013]. Only qualitative analyses were performed because of the variability of the interventions and outcome measures (e.g. small sample sizes, different outcome measures to assess white spot lesions, statistical analyses in some cases based on the tooth surfaces instead of the patient). Postorthodontic lesions are a desirable target for studies on remineralization strategies, as treating these lesions with concentrated fluoride agents will mineralize the surface but not the body of the lesion, thus allowing the arrested lesions (depending on their location) to remain as an esthetic concern over time [Beerens et al., 2010]. It is thought that the CPP-ACP mechanism of action allows ions to penetrate more deeply, leading to remineralization throughout the body of the lesion rather than just in the surface layer, resulting in improved esthetics. These studies tend to have relatively short intervention periods, as it is believed that the main regression of postorthodontic white spot lesions will likely take place immediately after debonding [Bröchner et al., 2011].

A 2013 systematic review [Tellez et al., 2013] of nonsurgical caries-preventive methods to arrest or reverse the progression of noncavitated caries lesions concluded that fluoride interventions have the most consistent benefit, while other strategies (including xylitol, chlorhexidine and CPP-ACP alone or in combination with fluoride) are very limited in number and in the majority of cases did not show a statistically significant reduction. The most recent systematic review on this topic included 8 studies (3-24 months) and concluded that there was a remineralizing effect of CPP-ACP compared to a placebo, but that the evidence regarding the clinical efficacy of CPP-ACP as a booster/supplement to fluoride-containing products is still unclear (the effect was not significantly different from that of fluorides) [Li et al., 2014].

\section{Amorphous Calcium Phosphate (ACP, ACFP)}

Two human clinical trials were identified in this study using ACP. In the first study ACFP was delivered in partitioned toothpaste to patients at high risk of caries due to decreased salivary flow following head and neck radiation. ACP is very unstable and may rapidly transform into a more stable phase such as hydroxyapatite (or fluorhydroxyapatite in the presence of fluoride). The test toothpaste was better than the control fluoride toothpaste in preventing root caries but not coronal caries [Papas et al., 2009]. In the second study a toothpaste with ACP was compared to a placebo toothpaste and to a fluoride toothpaste in high-caries-risk schoolchildren in India. Both the ACP and fluoride toothpastes did significantly better than the placebo [Rao et al., 2009].

$\mathrm{CaF}_{2}$

One study was identified testing addition of $\mathrm{CaF}_{2}$ to an $\mathrm{NaF}$ varnish compared to a NaF varnish only in Brazil. Over 4 weeks both varnishes performed similarly [Ferreira et al., 2009].

\section{Dicalcium Phosphate Dehydrate}

Two studies were identified testing dicalcium phosphate dehydrate in either a sodium fluoride or MFP toothpaste. Dicalcium phosphate dehydrate is commonly used as an abrasive, primarily used with MFP toothpastes. The dicalcium phosphate dehydrate toothpaste with $\mathrm{NaF}$ in a dual-tube toothpaste provided a superior anticaries efficacy compared to the control fluoride toothpaste [Boneta et al., 2001; Silva et al., 2001].

\section{Other Calcium-Based Strategies}

There were no clinical studies meeting the inclusion criteria in the current search to test the efficacy of Novamin, tricalcium phosphate, calcium carbonate and nanohydroxyapatite (a caries trial in Japanese schoolchildren was not included due to the publication time [Kani et al., 1989]), either alone or in combination with fluoride, in enhancing remineralization and preventing demineralization.

\section{Polyphenols}

Polyphenols (flavonoids, proanthocyanidins and others) are available in a variety of foods including fruits, vegetables, cereals, honey/propolis, olive oil, cocoa and
Fontana 
beverages such as tea (green, oolong-black, chamomile), wine and coffee. They have a wide range of biological activities, including antibacterial effects on cariogenic streptococci, related in part to their antioxidant action. There is some epidemiological evidence that the consumption of polyphenol-rich foods may significantly reduce the risk of some chronic conditions such as cardiovascular diseases, certain types of cancer and neurodegenerative disorders [Ferrazzano et al., 2011; Varoni et al., 2012]. However, the current search did not identify any clinical studies that met the inclusion criteria to support their use in the prevention of dental caries, or as boosters or alternatives to fluorides.

\section{Strategies Affecting the Biofilm}

Probiotics

Probiotics are live microorganisms that when administered in adequate amounts can provide a health benefit. The hypothesis is that probiotic strains interfere and/or inhibit other microorganisms, especially pathogens. Furthermore, a systemic modulation of immunological parameters is thought to also be a part of the interaction. Only 1 study fitting most of the search inclusion criteria, except that the vehicle was milk [Petersson et al., 2011], was identified in the current search and also in a recent systematic review [Twetman and Keller, 2012]. This fourarm parallel trial studied the effect of milk enriched with Lactobacillus rhamnosus on active root caries lesions over 15 months. All experimental groups resulted in increased root caries reversal/arrest compared to the group consuming regular control milk when assessed visually/tactilely and with the aid of electrical caries monitoring. The intervention was most effective in the probiotic/fluoride group, suggesting that probiotic bacteria might be considered as a booster or supplement to fluoride in preventing dental caries [Twetman and Keller, 2012].

\section{Xylitol}

Xylitol is a five-carbon sugar alcohol that has the same dietary sweetening property as sucrose. Good evidence supports that xylitol is noncariogenic, has a dose-/frequency-dependent effect on dental plaque/mutans streptococci and is safe. Even when the level/strength of highquality anticaries evidence is still limited, most recent systematic reviews have consistently concluded that the habitual use of sucrose-free xylitol or polyol combination chewing gum/lozenges are an effective adjunct in coronal caries prevention [Fontana and Gonzalez-Cabezas, 2012]. The current search resulted in 2 clinical studies that met the inclusion criteria and studied the effect of polyols in toothpastes. There were 2 additional studies that tested the effect of polyols in toothpastes but lacked a fluoride control arm, which is why they were excluded [Petersson et al., 1991; Chi et al., 2012]. In addition, there were 2 other excluded studies that compared the use of xylitol gum or chewable tablets to fluoride toothpaste or varnish in young children [Kovari et al., 2003; Alamoudi et al., 2012]. Regarding the included studies, Sintes et al. [1995] compared toothpaste with fluoride with and without $\mathrm{xy}$ litol. The study reported a $12.3 \%$ reduction in 3-year caries increment when using a sodium fluoride toothpaste $(1,100 \mathrm{ppm} F)$ in a silica base plus $10 \%$ xylitol. The second included study evaluated an MFP toothpaste $(1,100 \mathrm{ppm}$ F) in a dicalcium phosphate dihydrate base plus $10 \% \mathrm{xy}$ litol versus a control toothpaste without xylitol. A total of 3,394 children aged 7-12 years in Costa Rica participated in this double-blind randomized clinical trial. A single calibrated dentist performed all clinical examinations (no radiographs were included). After 30 months, the decayed filled surfaces (DFS) increment for the xylitol paste was 1.3 versus 1.51 for the control, and the decayed filled teeth (DFT) increment for the xylitol paste was 0.69 versus 0.81 for the control, which were statistically significantly different (a $13.9 \%$ reduction in DFS and a $14.8 \%$ reduction in DFT of the xylitol paste vs. the control [Sintes et al., 2002]). Milgrom et al. [2012] have stated that xylitol toothpaste studies in young children might require repeated use to maximize efficacy. In a systematic review Mickenautsch and Yengopal [2012] appraised the clinical evidence (12 prospective studies including a control arm) regarding the anticariogenic effect of xylitol compared to that of fluoride. They concluded that the addition of xylitol to existing fluoride regimes may be beneficial in the prevention of caries, but the included studies had limitations associated with potential risk of selection, as well as performance and attrition bias.

\section{Arginine}

The addition of arginine to toothpaste provides a potential anticaries benefit in three ways. Firstly, arginine may be metabolized by arginolytic plaque bacteria to produce a base which helps to neutralize acids formed by acidogenic bacteria in plaque [Wolff et al., 2013]. Secondly, the availability of arginine favors the presence of arginolytic bacteria helping to maintain a less cariogenic biofilm (prebiotic effect). Finally, the use of an insoluble calcium compound in the toothpaste base formulation helps to suppress the solubilization of tooth mineral when attacked by plaque acids [Kleinberg, 1999; Cummins, 2013]. Thus, this approach works at the biofilm level and acts as 
a supplement to fluoride in toothpaste, which in contrast acts on the tooth mineral to promote remineralization and help prevent demineralization. Eight clinical studies were identified in the current search. The initial studies that were conducted on arginine-containing products demonstrated the proof of principle that the introduction of arginine into the oral biofilm from either nonfluoride toothpaste (or through chewing arginine-containing candies, although this was excluded due to the type of vehicle in the present review [Acevedo et al., 2008]) had beneficial effects on reducing the incidence of dental caries [Acevedo et al., 2005]. In the case of the addition of arginine to nonfluoride toothpaste the anticaries benefit seen was similar to that derived from the control fluoride toothpaste.

More recently a series of clinical studies $(n=7)$ have tested the efficacy of the addition of $1.5 \%$ arginine to fluoride-containing toothpaste $(1,450 \mathrm{ppm} \mathrm{F})$ formulated in an insoluble calcium base; 3 of these studies considered the remineralization of initial enamel lesions over a 6-month period (table 2) monitored using quantitative light-induced fluorescence (QLF), 2 clinical studies assessed the ability of the formulation to remineralize primary root caries lesions over a period of 6 months, one 2 -year clinical study investigated the ability of the product to prevent cavity formation, and finally a 2 -year community-based study investigated the benefits of using an arginine-containing toothpaste in a school-based toothbrushing program. The 3 studies considering the remineralization of initial enamel lesions were all of a similar design (table 2). Children with initial caries lesions on the anterior teeth were recruited in schools and provided with randomly allocated toothpaste for use at home and also in a supervised brushing program instigated in their schools. Approximately 150 children per study group were recruited and closely monitored using QLF at baseline and after 3 and 6 months of using the products. In 2 of the studies [Yin et al., 2013a, b] the students used one of three toothpastes with either 1,450 ppm F with $1.5 \%$ arginine, $1,450 \mathrm{ppm} F$ without $1.5 \%$ arginine, or toothpaste without fluoride. In the third study [Srisilapanan et al., 2013] children used one of two toothpastes that contained either $1,450 \mathrm{ppm} F$ with $1.5 \%$ arginine or 1,450 ppm F without $1.5 \%$ arginine. The subject mean QLF metrics measured included lesion area (square millimeters), the percent loss of fluorescence compared to sound enamel $(\Delta \mathrm{F})$, which equates to lesion depth, and the product of the area and $\Delta \mathrm{F}$, which represents the lesion volume $(\Delta Q)$. In all cases $\Delta Q$ at 6 months was the primary outcome of the study. The subject mean baseline $\Delta \mathrm{Qs}$ for the 3 studies were very consistent (27.26-28.62). After 6 months the $\Delta \mathrm{Q}$ for children using the arginine-containing toothpaste ranged from 13.46 to 15.85 , representing $45-51 \%$ reductions in lesion volumes. For the children using toothpaste with fluoride alone, after 6 months the $\Delta$ Qs ranged from 17.99 to 20.35 , representing $29-34 \%$ reductions in lesion volumes. In the 2 studies in which children used the nonfluoride toothpastes the overall percentage reductions in lesion volume were 11 and 13\%. In all cases the differences between the fluoride toothpaste with arginine and the fluoride toothpaste without arginine were statistically significant in favor of the argininecontaining toothpaste. In addition, the subjects using the toothpaste containing fluoride alone had statistically significantly greater reductions in lesion volume during the 6 months of the study than those using the nonfluoride toothpaste.

The 2 studies (table 3 ) which assessed the ability of fluoride toothpaste containing arginine and fluoride to remineralize primary root caries were also of a similar design. Adults with 1 or more primary root caries lesions that were leathery or soft in texture at baseline were provided with toothpaste for use at home. After both 3 and 6 months of using of their randomly assigned product they were assessed to see whether the lesions had become hard (remineralized). In 1 of the 2 studies [Hu et al., 2013] the participants used one of three toothpastes containing either $1,450 \mathrm{ppm}$ F with $1.5 \%$ arginine, $1,450 \mathrm{ppm} F$ without $1.5 \%$ arginine, or toothpaste without fluoride. In the second study [Souza et al., 2013] the participants used one of two toothpastes that contained either $1,450 \mathrm{ppm} F$ with $1.5 \%$ arginine or $1,450 \mathrm{ppm} \mathrm{F}$ without $1.5 \%$ arginine. Overall, there were between 124 and 141 subjects completing the study in each of the study groups. In the first study [Hu et al., 2013], after 6 months $65.5 \%$ of the subjects had 1 or more primary root caries lesions becoming hard in the fluoride in the arginine group compared to $59.5 \%$ in the fluoride alone group and $25 \%$ in the nonfluoride group. Differences between all pair comparisons were statistically significant, with the arginine-containing toothpaste being superior to the fluoride alone toothpaste and both products being significantly better than the nonfluoride control. Remarkably, at the end of the 6-month study for the arginine-containing toothpaste only 1 subject (1.2\%) had 1 of more lesions that were leathery at baseline that became soft compared to $15.2 \%$ in the fluoride only group and $31.3 \%$ in the nonfluoride group. In the second study [Souza et al., 2013] after 6 months' use of the products $70.5 \%$ of the leathery lesions had become hard in the arginine group compared to
Fontana 
$58.1 \%$ in the group that used the toothpaste that only contained fluoride. Again, these differences were statistically significant.

The results of a 2-year clinical study that compared two toothpastes containing fluoride $(1,450 \mathrm{ppm} \mathrm{F})$ and $1.5 \%$ arginine with different abrasive systems (dicalcium phosphate or calcium carbonate) with fluoride toothpaste that did not contain arginine were reported by Kraivaphan et al. [2013]. The 6,000 participants, aged 6-12 years at baseline, were from a low-to-moderate caries risk population in Bangkok, Thailand. They were instructed to brush twice daily with their randomly assigned dentifrice. After 2 years, the two groups using the dentifrices containing $1.5 \%$ arginine, an insoluble $1,450 \mathrm{ppm} \mathrm{F}$ and a calcium compound had statistically significantly lower decayed, missing and filled teeth (DMFT) increments (21.0 and $17.7 \%$ reductions, respectively) and decayed, missing and filled surface (DMFS) increments (16.5 and 16.5\%) compared to the fluoride control dentifrice. The differences between the two groups using the arginine-containing toothpastes were not statistically significant.

Finally, a 2-year community-based study undertaken in Southern Thailand was reported by Petersen et al. [2015]. This assessed the caries-preventive benefit of an enhanced oral health promotion program, combined with a supervised toothbrushing and oral health education program in schools, using toothpaste containing $1,450 \mathrm{ppm} F$ and $1.5 \%$ arginine. A control group used fluoride toothpaste in a less well supervised program. A total of 15 southern Thailand schools and 3,706 preschool children were recruited. After 2 years' participation in the study the DMFT and DMFS increments for combined enamel and dentine lesions were 1.19 and 1.91 for the control group, and 1.04 and 1.59 for the intervention groups. These represent statistically significant 12.6 and $16.8 \%$ reductions in caries, respectively, compared to the control group. The DMFT and DMFS increments at the dentine threshold were 0.26 and 0.44 for the control group and 0.19 and 0.29 for the intervention group, representing statistically significant 26.9 and $34.1 \%$ reductions in caries incidence, respectively. As might be expected, the benefits were greater for the more cooperative schools, with up to a $40.9 \%$ reduction in caries for DMFS at the dentine threshold of diagnosis.

Taken as a whole, the included clinical studies, undertaken in a range of populations with different caries presentations, suggest that the addition of $1.5 \%$ arginine to toothpaste containing 1,450 ppm F (such as MFP) with an insoluble calcium base has the potential to significantly boost the performance of toothpaste with fluoride alone. However, it must be mentioned that all these studies have been performed by the group developing the arginine toothpaste, and independent studies would be welcomed.

\section{Conclusion}

Based on the current review, the amount of clinical evidence of the effectiveness for remineralization systems as boosters or alternatives to fluoride is limited. The current study did not identify evidence for any strategy to effectively be used as a substitute or alternative to fluoride for caries control, but identified some evidence derived from the use of microbial-related strategies (primarily from use of arginine combined with calcium) to support their potential use to boost the mechanism of action of fluoride. Thus, fluoride-based strategies remain the standard for caries prevention and management [Zero, 2009; Amaechi and van Loveren, 2013], with some limited evidence that boosting the effects of fluoride by the use of microbial-related strategies is a promising possibility. More long-term longitudinal studies, focusing in high-risk populations and confirmed by different research groups, will help confirm these findings over time.

\section{Acknowledgment}

Dr. Fontana acknowledges the support of Dr. Roger Ellwood from the Colgate Palmolive Company in the preparation of the section related to the incorporation of arginine into fluoride toothpaste.

\section{Disclosure Statement}

Dr. Fontana was funded to attend the conference by the Colgate Palmolive Company. She has no conflicts of interest to declare in relation to the manuscript.

\section{References}

Acevedo AM, Machado C, Rivera LE, Wolff M, Kleinberg I: The inhibitory effect of an arginine bicarbonate/calcium carbonate CaviStat-containing dentifrice on the development of dental caries in Venezuelan school children. J Clin Dent 2005;16:63-70.

Acevedo AM, Montero M, Rojas-Sanchez F, Machado C, Rivera LE, Wolff M, Kleinberg I: Clinical evaluation of the ability of CaviStat in a mint confection to inhibit the development of dental caries in children. J Clin Dent 2008;19:1-8. 
Alamoudi NM, Hanno AG, Masoud MI, Sabbagh HJ, Almushayt AS, Masoud IM: Effects of xylitol on salivary mutans streptococcus, plaque level, and caries activity in a group of Saudi mother-child pairs. An 18-month clinical trial. Saudi Med J 2012;33:186-192.

Amaechi BT, van Loveren C: Fluorides and nonfluoride remineralization systems. Monogr Oral Sci 2013;23:15-26.

Altenburger MJ, Gmeiner B, Hellwig E, Wrbas KT, Schirrmeister JF: The evaluation of fluorescence changes after application of casein phosphopeptides (CPP) and amorphous calcium phosphate (ACP) on early carious lesions. Am J Dent 2010;23:188-192.

Andersson A, Sköld-Larsson K, Hallgren A, Petersson LG, Twetman S: Effect of a dental cream containing amorphous cream phosphate complexes on white spot lesion regression assessed by laser fluorescence. Oral Health Prev Dent 2007;5:229-233.

Azarpazhooh A, Limeback H: Clinical efficacy of casein derivatives: a systematic review of the literature. J Am Dent Assoc 2008;139:915924.

Bader JD: Casein phosphopeptideamorphous calcium phosphate shows promise for preventing caries. Evid Based Dent 2010;11:11-12.

Bailey DL, Adams GG, Tsao CE, Hyslop A, Escobar K, Manton DJ, Reynolds EC, Morgan MV: Regression of postorthodontic lesions by a remineralizing cream. J Dent Res 2009;88: 1148-1153.

Beerens MW, van der Veen MH, van Beek H, ten Cate JM: Effects of casein phosphopeptide amorphous calcium fluoride phosphate paste on white spot lesions and dental plaque after orthodontic treatment: a 3-month follow-up. Eur J Oral Sci 2010;118:610-617.

Benson PE, Parkin N, Millett DT, Furness S, Germain P: Fluorides for the prevention of early tooth decay (demineralized white lesions) during fixed brace treatment. Cochrane Database Syst Rev 2013;12:CD003809.

Boneta AE, Neesmith A, Mankodi S, Berkowitz HJ, Sanchez I, Mostler K, Stewart B, Sintes J, De Vizio W, Petrone ME, Volpe AR, Zhang YP, McCool JJ, Bustillo E, Proskin HM: The enhanced anticaries efficacy of a sodium fluoride and dicalcium phosphate dehydrate dentifrice in a dual chambered tube. A 2-year clinical caries study on children in the United States of America. Am J Dent 2001;14:13A$17 \mathrm{~A}$.

Bröchner A, Christensen C, Kristensen B, Tranæus S, Karlsson L, Sonnesen L, Twetman S: Treatment of post-orthodontic white spot lesions with casein phosphopeptide-stabilized amorphous calcium phosphate. Clin Oral Investig 2011;15:369-373.

Chen H, Lium X, Dai J, Jiang Z, Guo T, Dingf Y: Effect of remineralizing agents on white spot lesions after orthodontic treatment: a systematic review. Am J Orthod Dentofacial Orthop 2013;143:376-382.
Chi DL, Tut O, Milgrom P: Cluster-randomized efficacy trial of a fluoride-xylitol toothpaste to prevent decay. J Dent Res 2012;91:3029.

Cochrane NJ, Cai F, Huq NI, Burrow MF, Reynolds EC: New approaches to enhanced remineralization of tooth enamel. J Dent Res 2010; 89:1187-1197.

Cummins D: The development and validation of a new technology, based upon $1.5 \%$ arginine, an insoluble calcium compound and fluoride, for everyday use in the prevention and treatment of dental caries. J Dent 2013;41:S1-S11.

Ferrazzano GF, Amato I, Ingenito A, Zarrelli A, Pinto G, Pollio A: Plant polyphenols and their anti-cariogenic properties: a review. Molecules 2011;16:1486-1507.

Ferreira JM, Aragao AK, Rosa AD, Sampaio FC, Menezes VA: Therapeutic effect of two fluoride varnishes on white spot lesions: a randomized clinical trial. Braz Oral Res 2009;23: 446-451.

Fontana M, Gonzalez-Cabezas C: Are we ready for definitive guidelines for xylitol use? Adv Dent Res 2012;24:123-128.

Hay KD, Thomson WM: A clinical trial of the anticaries efficacy of casein derivatives complexed with calcium phosphate in patients with salivary gland dysfunction. Oral Surg Oral Med Oral Pathol Oral Radiol Endod 2002;93:271-275.

Hu DY, Yin W, Li X, Feng Y, Zhang YP, Cummins D, Mateo LR, Ellwood RP: A clinical investigation of the efficacy of a dentifrice containing $1.5 \%$ arginine and $1,450 \mathrm{ppm}$ fluoride, as sodium monofluorophosphate in a calcium base, on primary root caries. J Clin Dent 2013; 24:A23-A31.

Huang GJ, Roloff-Chiang B, Mills BE, Shalchi S, Spiekerman C, Korpak AM, Starrett JL, Greenlee GM, Drangsholt RJ, Matunasj JC: Effectiveness of MI Paste Plus and PreviDent fluoride varnish for treatment of white spot lesions: a randomized controlled trial. Am J Orthod Dentofacial Orthop 2013;143:31-41.

Kani K, Kani M, Isozaki A, Shintani H, Ohashi T, Tokumoto T: Effect of apatite-containing dentifrices on dental caries in school children. J Dent Health 1989;19:104-109.

Kleinberg I: A new saliva-based anticaries composition. Dent Today 1999;18:98-103.

Kovari H, Pienihäkkinen K, Alanen P: Use of xylitol chewing gum in daycare centers: a follow-up study in Savonlinna, Finland. Acta Odontol Scand 2003;61:367-370.

Kraivaphan P, Amornchat C, Triratana T, Mateo LR, Ellwood R, Cummins D, DeVizio W, Zhang YP: Two-year clinical study of caries the efficacy of novel dentifrices containing $1.5 \%$ arginine, an insoluble calcium compound and 1,450 ppm fluoride. Caries Res 2013;47:582-590.
Li J, Xie X, Wang Y, Yin W, Antoun JS, Farella M, Mei L: Long-term remineralizing effect of casein phosphopeptideamorphous calcium phosphate (CPP-ACP) on early caries lesions in vivo: a systematic review. J Dent 2014;42: 769-777.

Marinho VCC, Higgins JPT, Logan S, Sheiham A: Fluoride gels for preventing dental caries in children and adolescents. Cochrane Database Syst Rev 2002;2:CD002280.

Marinho VCC, Higgins JPT, Logan S, Sheiham A: Fluoride mouthrinses for preventing dental caries in children and adolescents. Cochrane Database Syst Rev 2003a;3:CD002284.

Marinho VCC, Higgins JPT, Sheiham A, Logan S: Fluoride toothpastes for preventing dental caries in children and adolescents. Cochrane Database Syst Rev 2003b;1:CD002278.

Marinho VCC, Higgins JPT, Sheiham A, Logan S: Combinations of topical fluoride (toothpastes, mouthrinses, gels, varnishes) versus single topical fluoride for preventing dental caries in children and adolescents. Cochrane Database Syst Rev 2004a;1:CD002781.

Marinho VCC, Higgins JPT, Sheiham A, Logan S: One topical fluoride (toothpastes, or mouthrinses, or gels, or varnishes) versus another for preventing dental caries in children and adolescents. Cochrane Database Syst Rev 2004b;1:CD002780.

Mickenautsch S, Yengopal V: Anticariogenic effect of xylitol versus fluoride - a quantitative systematic review of clinical trials. Inter Dent J 2012;62:6-20.

Milgrom P, Söderling EM, Nelson S, Chi DL, Nakai Y: Clinical evidence for polyol efficacy. Adv Dent Res 2012;24:112-116.

Papas A, Russell D, Singh M, Stack K, Kent R, Triol C, Winston A: Double blind clinical trial of a remineralizing dentifrice in the prevention of caries in a radiation therapy population. Gerodontology 1999;16:2-10.

Petersen PE, Jurgensen N, Hintao J, Pithpornchaiyakul S, Thearmontree A, Hunsrisakhun J, Ellwood RP: School-based intervention for improving the oral health of children in southern Thailand. Community Dent Health 2015;44-51.

Petersson LG, Birkheb D, Gleerup A, Johansson $\mathrm{M}$, Jonsson G: Caries preventive effects of dentifrices containing various types and concentrations of fluorides and sugar alcohols. Caries Res 1991;25:74-79.

Petersson LG, Magnusson K, Hakestam U, Baigi A, Twetman S: Reversal of primary root caries lesions after daily intake of milk supplemented with fluoride and probiotic lactobacilli in older adults. Acta Odontol Scand 2011;69: 321-327.

Plonka KA, Pukallus ML, Holcombe TF, Barnett AG, Walsh LJ, Seow WK: Randomized controlled trial: a randomized controlled clinical trial comparing a remineralizing paste with an antibacterial gel to prevent early childhood caries. Pediatr Dent 2013;35:8-12. 
Rao SK, Bhat GS, Aradhya S, Devi A, Bhat M: Study of the efficacy of toothpaste containing casein phosphopeptide in the prevention of dental caries: a randomized controlled trial in 12- to 15-year-old high caries risk children in Bangalore, India. Caries Res 2009;43:430435.

Reynolds EC: Calcium phosphate-based remineralization systems: scientific evidence? Aust Dent J 2008;53:268-273.

Robertson MA, Kau CH, English JD, Lee RP, Powers J, Nguyen JT: MI Paste Plus to prevent demineralization in orthodontic patients: a prospective randomized controlled trial. Am J Orthod Dentofacial Orthop 2011;140:660668.

Silva MF, Melo EV, Stewart B, De Vizio W, Sintes JL, Petrone MF, Volpe AR, Zhang Y, McCool JJ, Proskin HM: The enhanced anticaries efficacy of a sodium fluoride and dicalcium phosphate dehydrate dentifrice in a dual chambered tube. A 2-year caries clinical study on children in Brazil. Am J Dent 2001;14: 19A-23A.

Sim CP, Wee J, Xu Y, Cheung YB, Soong YL, Manton DJ: Anticaries effect of CPP-ACP in irradiated nasopharyngeal carcinoma patients. Clin Oral Investig 2015;19:1005-1011.

Sintes JL, Elias-Boneta A, Stewart B, Volpe AR, Lovett J: Anticaries efficacy of a sodium monofluorophosphate dentifrice containing xylitol in a dicalcium phosphate dehydrate base. A 30-month caries clinical study in Costa Rica. Am J Dent 2002;15:215-219.

Sintes JL, Escalante C, Stewart B, McCool JJ, Garcia L, Volpe AR, Triol C: Enhanced anticaries efficacy of a $0.243 \%$ sodium fluoride $/ 10 \%$ xylitol/silica dentifrice: 3-year clinical results. Am J Dent 1995;8:231-235.
Sitthisettapong T, Phantumvanit P, Huebner C, Derouen T: Effect of CPP-ACP paste on dental caries in primary teeth: a randomized trial. J Dent Res 2012;91:847-852.

Souza MLR, Cury JA, Tenuta LMA, Zhang YP, Mateo LR, Cummins D, Ellwood RP: Comparing the efficacy of a dentifrice containing $1.5 \%$ arginine and $1,450 \mathrm{ppm}$ fluoride to a dentifrice containing $1,450 \mathrm{ppm}$ fluoride alone in the management of primary root caries. J Dent 2013;41:S35-S41.

Srisilapanan P, Korwanich N, Yin W, Chuensuwonkul C, Mateo LR, Zhang YP, Cummins D, Ellwood RP: Comparison of the efficacy of a dentifrice containing $1.5 \%$ arginine and 1,450 ppm fluoride to a dentifrice containing 1,450 ppm fluoride alone in the management of early coronal caries as assessed using quantitative light-induced fluorescence. J Dent 2013; 41:S29-S34.

Tellez M, Gomez J, Kaur S, Pretty IA, Ellwood R, Ismail AI: Nonsurgical management methods of noncavitated carious lesions. Community Dent Oral Epidemiol 2013;41:79-96.

ten Cate JM: Novel anticaries and remineralizing agents. Prospects for the future. J Dent Res 2012;91:813-815.

Twetman S, Keller MK: Probiotics for caries prevention and control. Adv Dent Res 2012;24: 98-102.

Varoni EM, Lodi G, Sardella A, Carrassi A, Iriti M: Plant polyphenols and oral health: old phytochemicals for new fields. Curr Med Chem 2012;19:1706-1720.

Walsh T, Worthington HV, Glenny AM, Appelbe $\mathrm{P}$, Marinho VCC, Shi X: Fluoride toothpastes of different concentrations for preventing dental caries in children and adolescents. Cochrane Database Syst Rev 2010;1:CD007868.
Wolff M, Corby P, Klaczany G, Santarpia P, Lavender S, Gittins E, Vandeven M, Cummins $\mathrm{D}$, Sullivan R: In vivo effects of a new dentifrice containing $1.5 \%$ arginine and $1,450 \mathrm{ppm}$ fluoride on plaque metabolism. J Clin Dent 2013;24:A45-A54.

Yazıcıoğlu O, Ulukapı $\mathrm{H}$ : The investigation of noninvasive techniques for treating early approximal carious lesions: an in vivo study. Int Dent J 2014;64:1-11.

Yengopal V, Mickenautsch S: Caries preventive effect of casein phosphopeptide-amorphous calcium phosphate (CPP-ACP): a meta-analysis. Acta Odontol Scand 2009;67:321-332.

Yetkiner A, Kara N, Ateș M, Ersin N, Ertuğrul F: Does casein phosphopeptide amorphous calcium phosphate provide remineralization on white spot lesions and inhibition of Streptococcus mutans? J Clin Pediatr Dent 2014;38: 302-306.

Yin W, Hu DY, Fan X, Feng Y, Zhang YP, Cummins D, Mateo LR, Pretty IA, Ellwood RP: A clinical investigation using quantitative lightinduced fluorescence (QLF) of the anticaries efficacy of a dentifrice containing $1.5 \%$ arginine and 1,450 ppm fluoride as sodium monofluorophosphate. J Clin Dent 2013a;24:A15-A22.

Yin W, Hu DY, Li X, Fan X, Zhang YP, Pretty IA, Mateo LR, Cummins D, Ellwood RP: The anti-caries efficacy of a dentifrice containing $1.5 \%$ arginine and $1,450 \mathrm{ppm}$ fluoride as sodium monofluorophosphate assessed using quantitative light-induced fluorescence (QLF). J Dent 2013b;41:S22-S28.

Zero DT: Recaldent - evidence for clinical activity. Adv Dent Res 2009;21:30-34. 